\title{
Eckpunkte für eine Strukturreform PFLEGE und TEILHABE
}

ROLF HOBERG,

THOMAS KLIE,

GERD KÜNZEL

Dr. Rolf Hoberg war nach seiner Tätigkeit im Sozialministerium BadenWürttemberg bis 2011 Vorstandsvorsitzender der AOK Baden-Württemberg; er ist Vorsitzender des Aussichtsrates des Wohlfahrtswerkes für BadenWürttemberg in Stuttgart

Prof. Dr. Thomas Klie ist Hochschullehrer für Öffentliches Recht und Verwaltungswissenschaft an der Evangelischen Hochschule Freiburg sowie an der Universität Wien/Klagenfurt

Gerd Künzel war bis 2012 Abteilungsleiter im Sozialministerium Brandenburg und ist Präsidiumsmitglied im DRK Landesverband Brandenburg in Potsdam und Mitglied der Bundesleitung der Gemeinschaft Wohlfahrtspflege und Sozialarbeit des DRK

\author{
Neben der dringlichen Verbesserung der \\ Finanzausstattung der Pflegeversicherung, um einen \\ erweiterten Begriff der Pflegebedürftigkeit, bessere \\ Personalschlüssel und einen Inflationsausgleich \\ zu ermöglichen, bestehen grundlegende \\ Strukturprobleme in der Pflegesicherung. Entlang der \\ drei Reformperspektiven: Unterscheidung von cure \\ und care bei den Pflegeaufgaben, Gleichbehandlung \\ von Pflegebedürftigkeit und Behinderung sowie \\ Stärkung der kommunalen Gestaltungsspielräume \\ bei der Sicherung von Pflege und Teilhabe \\ wird eine Strukturreform vorgeschlagen.
}

\section{Pflege und Teilhabe sozialpolitisch neu ordnen}

Die 18. Legislaturperiode könnte eine Legislatur der Pflege- und Teilhabereform werden. Die Einführung eines neuen Pflegebedürftigkeitsbegriffes steht ebenso an wie der Verlagerung von Teilen der Eingliederungshilfe auf die Bundesebene. Zudem ist eine breite Diskussion entbrannt - und dies über die Parteigrenzen hinweg - wie wir aus einem verengten Verständnis von Pflege heraustreten , wie die Kommunen in ihrer Rolle gestärkt werden und insgesamt ein Innovationsklima in der Gestaltung von Pflegeaufgaben in der Gesellschaft geschaffen werden kann - jenseits von Pflegedokumentation und Pflegenoten.

Ein neuer Pflegebedürftigkeitsbegriff ist seit langem in der Diskussion, alternative Ausgestaltungen sind mit ihren Chancen für die Menschen und Folgen für die notwendige Finanzausstattung durchgerechnet - allein es fehlte bisher die politische Kraft zur Umsetzung.

Einigkeit besteht auch darüber, dass die Personalsituation in der Pflege verbessert werden muss. Für Pflegebedürftige wie für Pflegende sind sowohl im ambulanten wie im stationären Bereich sind die gegenwärtigen Grundlagen für die Finanzierung des Personals ein Hindernis für gute Pflege und attraktive Berufsbedingungen. Nur zusammen mit einer besseren Vergütung wird es gelingen in einem sich rasch verengenden Arbeitsmarkt genügend Menschen für den Pflegeberuf zu gewinnen.

Ungelöst ist auch das Problem der schleichenden Entwertung der Pflegeversicherungsleistungen durch die branchenspezifische Inflation. Steigende Zahlen der Sozialhilfeabhängigkeit belegen diese Entwicklung. Hier ist sowohl für die Vergangenheit als auch perspektivisch eine Korrektur erforderlich.

Diese bekannten dringlichen Problemanzeigen sind gut dokumentiert. Lösungsvorschläge liegen vor. Die hier entwickelten Reformvorschläge gehen aber von der Einsicht aus, dass selbst bei Umsetzung aller bisher genannten Verbesserungen gravierende Strukturprobleme der Pflegesicherung bestehen bleiben, für die Lösungen gefunden werden müssen.

In der vergangenen Legislaturperiode ist es nicht gelungen, eine zukunftssichere und nachhaltige Pflegereform auf den 
Weg zu bringen. Bei aller Enttäuschung hierüber bietet das nun notwendige weitere Ringen um eine große Pflegereform auch die Chance, die Weichen für eine Strukturreform PFLEGE und TEILHABE zu stellen. In einem zweijährigen Diskussionsprozess, an dem Experten aus allen Ebenen und Sektoren der Pflege- und Teilhabepolitik beteiligt waren, wurden die hier vorgestellten Eckpunkte für eine Strukturreform erarbeitet. ${ }^{1}$ Sie ordnen auch das Verhältnis zwischen ambulanter und stationärer Pflege neu.

\section{Die zentralen Reformperspektiven}

Die Strukturreform verfolgt zentral das Anliegen, die dysfunktionalen Schnittstellenprobleme zwischen den Leistungsbereichen Gesundheit, Pflege und Teilhabe zu lösen und ein funktionierendes Koordinierungssystem zu finden, das auch Formen ambulanter und stationärer Versorgung durchlässiger gestaltet.

\subsection{Neue Ordnung: CURE und CARE}

Als tragender Grundgedanke für eine Strukturreform bietet sich eine Differenzierung von Pflege an. Die Pflegeversicherung versteht heute unter Sicherung der Pflege

- Pflegeleistungen,

- hauswirtschaftliche Leistungen,

n soziale Betreuungsleistungen.

Vorgeschlagen wird, den Inhalt dessen, was in Deutschland unter Pflege verstanden und nach den leistungsrechtlichen Vorschriften an Leistungen gewährt wird, in CURE und CARE zu unterscheiden. Diese Empfehlung findet sich bereits im 6. Altenbericht und aus der letzten Legislaturperiode im Endbericht zum Zukunftsdialog der Bundeskanzlerin. Durch eine Differenzierung in CURE und CARE sollen ein ganzheitliches Menschenbild und der Anspruch auf umfassende und koordinierte Unterstützung von auf Pflege angewiesenen Menschen befördert werden ohne alle Leistungen und Hilfen unter die Regie und den Vorbehalt der Fachpflege zu stellen.

Als CURE werden medizinische, pflegerische und therapeutische Maßnahmen verstanden, die von professionellen Akteuren im Gesundheitswesen zur Prävention, Kuration, Rehabilitation und Palliation angeboten, geleistet und verantwortet werden.

$\mathrm{Zu}$ CARE zählen alle Formen der Sorge und Versorgung, die für den Lebensalltag erforderlich sind - personenbezogene Leistungen zur unterstützender Alltagsgestaltung, hauswirtschaftlichen Basisversorgung, Grundpflege und Förderung der sozialen Teilhabe.

Die Steuerung von CURE und das Zusammenwirken mit CARE übernehmen die im CURE-Sektor Tätigen: Medizin und Fachpflege verantworten Diagnostik und Therapie, erarbeiten Behandlungs- und Pflegepläne und steuern den Behandlungs- und Pflegeprozess. Dabei beziehen sie je nach Ausgangssituation und Verlauf sowohl Angehörige, Freunde und Nachbarn des Pflegebedürftigen in die Versorgung mit ein als auch berufliche oder ehrenamtlich Helfende sowie Assistenzkräfte. Die Aufgaben der CARE-Akteure liegen demgegenüber in der Alltagsgestaltung, Organisation des Haushalts, Sicherung der Sozialkontakte etc., daheim und im Heim.

Eine auf der Unterscheidung von CURE und CARE basierende Abgrenzung von Leistungen und Zuständigkeiten ist Basis für eine grundlegende Reform.

\subsection{Neuordnung des Leistungsrechts}

Kernelement der Strukturreform ist eine Neuordnung des Leistungsrechts, die der Differenzierung in CURE- und CARELeistungen folgt. Danach werden

- CURE-Leistungen der Gesetzlichen Krankenversicherung (GKV) zugeordnet

- CARE-Leistungen der Pflegeversicherung und der Eingliederungshilfe bzw. einem Bundesleistungsgesetz Teilhabe.

\section{Neue Finanzierungstechnik}

Wegen der unterschiedlichen Zwecke und Steuerung der Leistungen für CURE und CARE ist eine neue Finanzierungstechnik gefragt:

- Für CURE: Sachleistungsprinzip mit Vollfinanzierung, ergänzt durch die Finanzierung von Leistungen zur Steuerung (medizinisch-pflegerische Versorgungs- und Hilfeplanung, Anleitung, Monitoring, Aushandlung).

- Für CARE: Abschaffung des Sachleistungsprinzips in der bisherigen Ausprägung. Leistungen müssen

1 Langfassung mit einer ökonomischen Folgeabschätzung: Hoberg, Klie, Künzel (2013): Strukturreform Pflege und Teilhabe. Ein Politikentwurf für eine nachhaltige Sicherung von Pflege und Teilhabe, Freiburg, FEL-Verlag; als pdf unter: www.agp-freiburg.de 
individuell und flexibel eingesetzt werden können und die Kosten zur Organisation der Alltagsgestaltung decken. Ein hoher Unterstützungsbedarf darf nicht automatisch zur Sozialhilfeabhängigkeit führen und muss daher leistungsrechtlich besser ausgestattet werden.

Ein Bundesleistungsgesetz ergänzt die Pflegeversicherung und reduziert die Sozialhilfe. Dies muss mit einer Reform des Leistungsrechts der Teilhabe abgestimmt werden.

\subsection{Integrierte Steuerung}

Ein Hilfesystem muss den Defiziten begegnen, die seine Effizienz unterlaufen. Die Mängel, um die es derzeit in der Praxis geht, zeigen sich in den Bereichen Aufmerksamkeit (für den Pflegeverlauf), Abklärung und Koordination. CURE und CARE brauchen daher leistungsträgerübergreifend sowohl ein neues Begutachtungswesen als auch eine Infrastruktur für den Aufbau von Care - und Case Management. Um die strukturellen Probleme der Pflegestützpunkte zu überwinden, werden Servicestellen für Pflege und Teilhabe mit einer Vermittlungs- und Weiterleitungsfunktion eingerichtet, die je nach Konstellation und Bedarf auf ein Assessment hinwirken. Für eine effektive Steuerung ist die Bündelung im Sozialraum Kommune erforderlich.

\section{Das Reformpaket}

\subsection{Mehr Prävention und Rehabilitation, Vernetzung und Flexibilität}

Um die Reform verwirklichen zu können, wird folgendes Maßnahmenpaket vorgeschlagen:

\section{Bessere rechtliche Integration der Pflege}

Integration der Pflege in das SGB I und der Pflegeversicherung in das SGB IX Die Pflege insgesamt wird übergreifend im SGB I beschrieben. Pflegeleistungen werden die 5. Leistungsgruppe der Teilhabeleistungen im SGB IX. Die Pflegeversicherung wird Rehaträger für die pflegerischen CARE-Leistungen und damit verbindlich in die (weiterzuentwickelnden) Kooperationsvorschriften des SGB IX eingebunden.

\section{Behandlungspflege unabhängig vom Leistungsort}

Die Behandlungspflege wird auch im stationären Bereich zur Leistung der GKV. Durch die Trennung von CURE und CARE wird das Interesse der GKV an Prävention und Rehabilitation auf die stationäre Pflege ausgedehnt. Bürokratischer Abgrenzungsaufwand zwischen ambulanter und stationärer Behandlungspflege entfällt damit. Dienste können übergreifend von einem Träger organisiert werden. Das steigert die Flexibilität und Produktivität.

Die Leistungsverschiebung von der Pflegeversicherung in die GKV kann beitragssatz- und wettbewerbsneutral gestaltet werden. In der Pflegeversicherung wird aus der Leistung für stationäre Pflege ein normierter Betrag herausgelöst und für alle stationär Versorgten als Gesamtbetrag in den Gesundheitsfonds überführt. Dort werden die Beträge auf die Krankenkassen morbiditätsentsprechend verteilt. Der im Einzelfall höhere Leistungsbedarf ist von der jeweiligen Krankenkasse zu tragen.

Wenn die Behandlungspflege von der GKV getragen wird, können die Leistungen für stationäre Pflege in der Pflegeversicherung ohne Schlechterstellung des Einzelnen um genau diesen Betrag gesenkt werden. Damit ergibt sich eine Neubestimmung der Leistungshöhe zwischen dem stationären und ambulanten Bereich. Dies reduziert den Fehlanreiz zugunsten stationärer Pflege.

\section{Umwandlung in Budgets}

Die als Sachleistung ausgestalteten Leistungsansprüche werden wertgleich in Geldbudgets (Sachleistungsbudgets) umgewandelt. Diese erlauben es Pflegebedürftigen, frei zwischen (lizenzierten, qualitätsgesicherten) Dienstleistern zu wählen. Die Höhe des Budgets wird so ausgestaltet, dass die Eigenbeteiligung bei zunehmendem Hilfebedarf prinzipiell gleich bleibt.

Die Leistungsform des persönlichen Budgets soll auch für Leistungen der Pflegeversicherung geöffnet werden.

Die Möglichkeit, die Gesamtleistung der Pflegeversicherung bei Bedarf für bestimmte Leistungskombinationen angemessen zu erhöhen, soll erhalten bleiben.

Die unterschiedliche Höhe von Pflegegeldleistungen und Pflegesachleistungen bleibt unberührt.

\section{Steuerung des Pflegeprozesses als Leistung der GKV}

Für eine wirkungsvolle Verschränkung der Pflege in CURE und CARE ist es erforderlich, die Steuerung des Pflegeprozesses bei den Fachpflegekräften zu konzentrieren und damit als eigenständige Leistung der GKV abzubilden.

\section{Regie als Zuschlagsleistung für integrierten Schutz}

Stationäre Versorgung gewährleistet Begleitung, Koordination und Schutz rund um die Uhr. Um flexible Versorgungsarrangements zu fördern, wird diese Besonderheit - bei vergleichbarer Versorgungssicherheit - zur eigenständigen Regieleistung und sowohl stationär als auch ambulant bei Sachleistungen abrechenbar gemacht.

\section{(Pflege)Hilfsmittel- Versorgung angleichen}

Die heute unterschiedliche Bereitstellung von (Pflege-)Hilfsmitteln in der stationären und ambulanten Pflege beeinträchtigt die Entwicklung neuer Versorgungformen. Für durchgängige Strukturen ist eine Angleichung unter Einbeziehung von Kranken- und Pflegeversicherung erforderlich.

\subsection{Bessere Beratungsinfrastruktur}

\section{Servicestelle Pflege und Teilhabe}

Das Informationsangebot heutiger Pflegeberatung und der Pflegestützpunkte ist grundsätzlich zu sehr auf den Leistungsbereich der Pflegeversicherung verengt. Die Aufgaben der Pflegestützpunkte und Teilaufgaben der gemeinsamen Servicestellen werden in einer Servicestelle Pflege und Teilhabe zusammengefasst - ggf. unter Einbeziehung der Pflegeberatung.

Hilfe- und pflegebedürftige Menschen haben gegenüber der Servicestelle Pflege und Teilhabe ein Antragsrecht auf Fallabklärung.

Hausarzt, Gesundheitszentrum, Krankenhaus, Quartiersmanagement und MDK vermitteln die Klienten an die Servicestelle Pflege und Teilhabe. Diese kann im Auftrag des Klienten gegenüber Beratungs- und Assessment-Agenturen (MDK, Gesundheitsamt) und Sozialleistungsträgern initiativ werden und ein Case Management vermitteln. 


\section{Gemeinsame Assessment-Agentur}

Um die Leistungsansprüche einer Person umfassend zu klären und ihre Versorgung $\mathrm{zu}$ planen und $\mathrm{zu}$ koordinieren, bedarf es einer weiteren und neu zu schaffenden Einrichtung: eine übergreifende Assessmentagentur auf Landesebene für Pflege und Teilhabe. Hier wird der Bedarf an Pflege und Rehabilitation ebenso festgestellt wie bei wesentlich Behinderten der Bedarf an Teilhabe am Arbeitsleben. In der (ggf. virtuellen) Assessmentagentur wirken MDK, die Bundesagentur für Arbeit, die Rentenversicherung und die kommunalen Arbeitsgemeinschaften zusammen.

\subsection{Bessere Koordination und Kooperation im Einzelfall}

\section{Budgets für Care - und Case Management}

Zur Förderung des vom Gesetzgeber mit der Pflegeberatung intendierten Care und Case Managements wird den Servicestellen Pflege und Teilhabe ein Budget von 20.000 Euro zu Lasten der GKV zugeordnet. Hieraus kann für komplexe Versorgungsfälle ein ggf. von Dritten durchzuführendes Case Management finanziert werden.

\section{Individuelle Piloten zur Fallbegleitung}

Die Begleitung des Einzelfalls ist eine Aufgabe, die sowohl die Servicestelle als auch die Assessmentagentur überfordert. Eine individuell verantwortliche Person (für die es Beispiele im CUREBereich mit der Spezialisierten Ambulanten Palliativen Versorgung (SAPV), bei einzelnen Krankenkassen als Modell des Patientenbegleiters und im CAREBereich als Modell des Pflegebegleiters oder Koordinators gibt), wird als Lösung gesehen, jeweils ergänzt um die Aufgaben der rechtlichen Betreuung oder Assistenz.

\section{Integrierte Versorgung: Überleitung in die nachstationäre Pflege verbessern}

Die deutlich kürzere Verweildauer im Krankenhaus verstärkt bei pflegebedürftigen Menschen und ihren Angehörigen den Druck, in kurzer Zeit zu entscheiden, wo und wie es nach dem Klinikaufenthalt pflegerisch weitergehen soll - zu Hause oder in einem Pflegeheim. Damit hier sorgfältiger geprüft, geplant und entschieden werden kann, wird vorgeschlagen: Durch weiterzuentwickelnde integrierte Versorgungsverträge der Kranken- und Pflegekassen mit Krankenhäusern, Ärzten, ambulanten Diensten, stationären Einrichtungen, Hilfsmittellieferanten und Apotheken wird die Überleitung vom Krankenhaus in die nachstationäre Versorgung auf Kreisebene verbessert.

\subsection{Sozialräumliche Orientierung: Kommunale Federführung als Grundprinzip}

Für Kommunen, Krankenkassen und Pflegeversicherung muss eine neue Kooperationsbasis gefunden werden, die einerseits die Zuständigkeiten der Sozialversicherung wahrt, andererseits aber den Kommunen mehr Einfluss- und Gestaltungsmöglichkeit bei der Umsetzung ihres Auftrags zur lokalen Daseinsvorsorge sichert.

Auf institutioneller Ebene müssen Beratung und Koordination in der Region (Kreis- und Landesebene) zusammengeführt und in die kommunale Daseinsvorsorge als Pflichtaufgabe eingebettet werden, damit die CURE- und CARE-Aufgaben übergreifend bearbeitet werden. Nur in kommunaler Verantwortung können die widersprüchlichen Steuerungen von wettbewerbsorientierter Krankenversicherung, einheitlich und gemeinsam handelnder Sozialer Pflegeversicherung, wettbewerbsorientierten Leistungserbringern und einheitlichen Fürsorgeleistungen überwunden werden.

Die kommunale Daseinsvorsorge beginnt mit der kommunalen Sozialplanung u.a. in der Bauleitplanung und Wohnraumversorgung, im Nahverkehr, in zivilgesellschaftlichen Netzwerken und setzt sich fort in der sozialräumlichen Koordination in Quartieren und Stadtteilen, bei der alle Lebensbereiche und Infrastrukturen in ihrem Zusammenhang gebündelt werden können.

Unterstützt werden muss dies durch Abstimmungen auf Landesebene im Rahmen der Zuständigkeiten bei Planung und Förderungen, zum Beispiel der Landeskrankenhausplanung, der Landespflegeplanung (u.a. Pflegestützpunkte), der Koordination von Freiwilligendiensten sowie im Rahmen der Stadtund Dorfentwicklung. Die kommunale Federführung muss bei ausbleibenden
Selbstverwaltungslösungen als Auffangzuständigkeit geschaffen werden.

\subsection{Pflege als Teilhabeleistung und Bundesleistungsgesetz}

Durch die Einführung des neuen Pflegebedürftigkeitsbegriffs ist Pflege als eine Teilhabeleistung anzusehen, die ohne Beschränkung auf die Versicherungsleistungen im SGB I und SGB XI verankert werden muss. Die bei der Umsetzung des Fiskalpakts getroffene Verabredung, die Eingliederungshilfe in ein neues Bundesleistungsgesetz zu überführen, sollte dazu genutzt werden, ein übergreifendes Teilhabegesetz zu schaffen, das nach Art und Schwere der Behinderung gestaffelte Teilhabeleistungen des Bundes vorsieht, die im Sinne eines Nachteilsausgleichs nicht nachrangig sind. Es sollte auch den Teilleistungscharakter des SGB XI ergänzen, die Ungleichbehandlung behinderter Pflegebedürftiger beenden und eine stimmige Verknüpfung mit den Teilhabeleistungen der Eingliederungshilfe ermöglichen.

\subsection{Qualität - an Personen statt an Einrichtungen orientieren}

Die Strukturreform macht auch den Weg frei zu einem Paradigmenwechsel bei der Qualitätssicherung. Wenn Menschen mit Pflege- und Hilfebedarf verlässlich assessmentgestützt begleitet werden und ihr Anspruch auf Beratung und Case Management sichergestellt ist, lassen sich die bisher vornehmlich institutionell ausgerichteten Aktivitäten zur Qualitätssicherung zu Gunsten einer personenbezogenen Qualitätssicherung zurücknehmen. Dieser Prozess müsste auch durch den neuen Pflegebedürftigkeitsbegriff unterstützt werden.

Zur pflegerischen Qualitätssicherung gehören im Wesentlichen die Evaluation des Hilfe- und Pflegeprozesses sowie die Sicherung der Selbstbestimmung des hilfebedürftigen Menschen und seiner Präferenzen. Auf umfassende Prüfungen von Heimen bezogen auf die pflegerische Qualität könnte weitgehend verzichten werden

Der CURE-Bereich unterliegt den Qualitätsmechanismen des SGB V. Für den CARE-Bereich bleiben Qualitätsvorgaben ordnungsrechtlicher Art sowie für die zugelassenen oder zertifizierten Dienste geltende Vorgaben hinsicht- 
lich der Struktur- und Prozessqualität verbindlich. Bei der Leistungsform des persönlichen Budgets werden die Zielvereinbarungen zu den entscheidenden Instrumenten der Qualitätssicherung.

\section{Kosten und Finanzierung}

Die Kosten der einzelnen Reformvorschläge sind überschaubar und gegenfinanziert. Als Kostenpositionen werden veranschlagt:

Pflegeprozesssteuerung

Regiekosten

Krankenhaus-Entlass-

management

Case Management

Fallunterstützung durch

„Piloten“

Versorgung mit

Hilfsmitteln

Servicestellen Teilhabe

und Pflege

Gemeinsame

Assessmentagentur

Kommunale Hilfe-

planung

57 Mio. € 45 Mio. $€$

7 Mio. $€$

10 Mio. $€$

108 Mio. $€$

bis zu 160 Mio. $€$

Org. Änderung

Org. Änderung

Org. Änderung

Dem stehen Einsparungen insbesondere durch den Abbau von Bürokratiekosten bei Übergang von einer institutionenbezogenen zur personenbezogenen Qualitätssicherung gegenüber, die mit $10 \%$ des Aufwandes für Dokumentation und Leistungsnachweise von rd. 2.000 Mio. $€$ erwartet werden.

Weitere positive Kosteneffekte der besseren Durchlässigkeit der Versorgungsbereiche, der Vermeidung von Krankenhauseinweisungen, Vermeidung von Fehl- und Unterversorgung, zielgenauere Hilfen, Mobilisierung zivilgesellschaftlicher Unterstützung sind absehbar.

Per Saldo sind aus den o.g. Reformvorschlägen keine spürbaren Zusatzbelastungen zu erwarten, aber eine Ergebnisverbesserung in Pflege und Teilhabe.

Zwei große Bereiche bleiben: die Verlagerung der Behandlungspflege in stationären Einrichtungen vom SGB XI ins SGB V und die Aufhebung der Leistungsbegrenzung für Behinderte in stationären Einrichtungen bei der Pflegeversicherung.

Bei der Behandlungspflege konnte im Rahmen einer ergänzenden Studie gezeigt werden, dass eine kosten-, leistungs- und beitragsneutrale Lösung machbar ist, die durch einen eine Anpassung des Risikostrukturausgleichs für die Krankenkassen ohne Wettbewerbsnachteile bleibt, auch wenn es sich um ein Finanzvolumen von immerhin 1,8 Mrd.€ handelt. ${ }^{2}$

Die Leistungsangleichung für Behinderte in stationären Einrichtungen bei der Pflegeversicherung kann bei gleichzeitiger Einführung eines Teilhabegeldes grundsätzlich durch eine Anrechnung auf Leistungen der Pflegeversicherung gegenfinanziert werden. Die Belastung im Einzelfall bedarf der sorgfältigen Erwägung, aber das Gesamtvolumen von ca. 1,4 Mrd. $€$ ist auch nach Einführung des neuen Pflegebedürftigkeitsbegriff darstellbar.

\section{Ausblick}

Die hier unterbreiteten Vorschläge für eine Strukturreform Pflege und Teilhabe stehen in engem inhaltlichen Bezug zu den Arbeiten aus dem Projektzusammenhang SONG („Soziales neu gestalten“), den Empfehlungen des Positionspapiers, das Kuratorium Deutsche Altershilfe und die Friedrich - Ebert - Stiftung „Gute Pflege vor Ort - Das Recht auf ein eigenständiges Leben im Alter" sowie den Diskussionen um ein regionales Pflegebudget, das als Projekt von der Bertelsmann - Stiftung gefördert und von der PROGNOS AG entwickelt wird. All diese weitgespannten Überlegungen dokumentieren die Einsicht in die Notwendigkeit grundlegender Reformen zur Sicherung der Pflege und Teilhabe. Sie konkurrieren nicht miteinander, sondern setzen unterschiedliche Akzente.

In Verlauf der Verhandlungen zu einem Koalitionsvertrag für die 18. Legislaturperiode haben sich die Akteure in einem gemeinsamen Appell an die Verhandlungspartner gewandt, um diese grundlegende Reform voranzubringen.

Betrachtet man den vorliegenden Koalitionsvertrag (Stand 29.Nov. 2013) so enthält dieser viele Absichtserklärungen, die mit den hier eingebrachten Vorschlägen korrespondieren. Wesentlich ist die wenn auch nur schrittweise - vorgesehene Verbesserung der Finanzausstattung über eine Anhebung der Beiträge. Damit können die beabsichtigte Leistungsausweitung, die zusätzlichen Betreuungskräfte sowie der neue Pflegebedürftigkeitsbegriff in großen Teilen umgesetzt werden. Die Leistungsanpassung an die Inflation erst ab 2015 korrigiert den eingetretenen Realverlust nicht. Für die Gleichbehandlung von Pflegebedürftigen und Behinderten bietet die vorgesehene Einführung eines Teilhabegeldes eine Chance. Der für die Pflegeversicherung formulierte Abwehrsatz gegen Kostenverlagerung von anderen Leistungsträgern unterstreicht die Notwendigkeit, in einem Gesamtkonzept zusammen mit den inhaltlichen Reformen die finanziellen Belastungen zwischen Sozialversicherung und Steuersystem auszubalancieren. Wir haben dazu einen Vorschlag formuliert. Inwieweit das Potential der vorgeschlagenen systematischen Unterscheidung von cure und care bereits im politischen Dialog erkannt wurde und aufgegriffen wird, bleibt abzuwarten. Immerhin enthält die Koalitionsvereinbarung einen Prüfauftrag zum Verhältnis von SGB XI und V-allerdings in beide Richtungen. Die Stärkung der kommunalen Gestaltungsmöglichkeiten in der Pflegesicherung wird als Absicht aufgegriffen, bleibt aber konkretisierungsbedürftig. Dabei ist die Rolle von Kommunen und Sozialversicherung so neu zu bestimmen, dass die Verantwortung für die Daseinsvorsorge zusammen mit den Kranken- und Pflegekassen wirksam wahrgenommen werden kann. Der Vorrang von Prävention und Rehabilitation in der Pflege wird als Zielsetzung aufgenommen, die Umsetzungsvorschläge hierzu waren im Verlauf der Koalitionsverhandlungen aber schon hilfreich konkreter (Arbeitsgruppe Gesundheit Stand 18. Nov. 2013). Die als Problem erkannte zu schnelle Verlegung vom Krankenhaus ins Pflegeheim wird in den Krankenkassen und den Krankenhäusern zur Lösung zugewiesen. Der Ausbau der Beratungsleistungen für die Pflegebedürftigen sollte verbunden werden mit einer konsequenten Verschränkung mit den jeweiligen kommunalen Beratungsstrukturen nur das würde - auch nach den Erfahrungen mit der Pflegeberatung und den Pflegestützpunkten - für die Pflegebedürftigen und ihre Angehörigen Sinn machen. Die Erweiterung der Rolle von Pflegekräften wird weiterhin nur zögerlich angegangen.

Insgesamt bietet der Koalitionsvertrag mit seinen absichtsvollen Formulierungen genügend Perspektive, um im Rahmen der gesetzlichen Umsetzung die vorliegenden Vorschläge zu einer Strukturreform Pflege und Teilhabe aufzugreifen.

2 Rothgang, Müller (2013): Ökonomische Expertise zur Verlagerung der medizinischen Behandlungspflege von der Pflegeversicherung in die Krankenversicherung, Bremen) 